\title{
HPV L1 detection discriminates cervical precancer from transient HPV infection: a prospective international multicenter study
}

Grit Mehlhorn ${ }^{1}$, Ellen Obermann ${ }^{2}$, Giovanni Negri ${ }^{3}$, Lukas Bubendorf ${ }^{2}$, Christine Mian ${ }^{3}$, Martin Koch ${ }^{1}$, Heinz Sander ${ }^{4}$, Birgit Simm ${ }^{4}$ Markus Lütge ${ }^{5}$, Zsuzsanna Bánrévi ${ }^{5, *}$, Anke Weiss ${ }^{6, * *}$, Gieri Cathomas ${ }^{7}$, Ralf Hilfrich ${ }^{8}$, Matthias Wilhelm Beckmann ${ }^{9}$ and Henrik Griesser ${ }^{10}$

${ }^{1}$ Department of Obstetrics and Gynecology, University Hospital Erlangen, Erlangen, Germany; ${ }^{2}$ Institute of Pathology, University Hospital Basel, Basel, Switzerland; ${ }^{3}$ Institute of Pathology, Central Hospital Bolzano, Bolzano, Italy; ${ }^{4}$ Cytology Laboratory Einbeck, Einbeck, Germany; ${ }^{5}$ Cytology Laboratory, Salzgitter Bad, Germany; ${ }^{6}$ Center of Pathology and Cytodiagnostics, Cologne, Germany; ${ }^{7}$ Kantonal Institut of Pathology, Liestal, Switzerland; ${ }^{8}$ Cytoimmun Diagnostics GmbH, Pirmasens, Germany; ${ }^{9}$ Department of Obstetrics and Gynecology, University Hospital Erlangen, Erlangen, Comprehensive Cancer Center Erlangen-Nürnberg, Germany and ${ }^{10}$ Department of Pathology and Cytodiagnostics, Laboratory Medicine Cologne Wisplinghoff and Colleagues, Cologne, Germany

The benefits of cytology-based cervical cancer screening programs in reducing morbidity and mortality are well recognized. Especially, overtreatment of human papillomavirus (HPV) high-risk positive early dysplastic lesions may have a negative impact on reproductive outcomes for fertile women. To optimize the clinical management an objective standard is needed to distinguish precancer that requires treatment, from spontaneously resolving HPV infections. In the current study, we examined the prognostic relevance of HPV-L1 capsid protein analysis with Cytoactiv in an international prospective multicenter study including 908 HPV high-risk positive early dysplastic lesions (LSIL/HSIL) during a follow-up period of 54 months. The clinical end points of the study were histologically confirmed CIN3 + as progression, CIN1/2 for stable disease and repeated negative Pap smears as spontaneous clinical remission. The difference of the clinical outcome of HPV-L1-negative and HPV-L1-positive cases was statistically highly significant $(P$-value $<0.0001)$ independent of the classification as mild dysplasia (LSIL) and moderate dysplasia (HSIL). Of the HPV-L1-negative HPV high-risk positive $\mathrm{mild} / \mathrm{moderate}$ dysplasias $84 \%$ progressed to CIN3, as compared with only $20 \%$ of the HPV-L1-positive cases. The data from our study show that HPV-L1 detection allows to identify transient HPV infections and precancerous lesions within the group of HPV high-risk positive early dysplastic lesions. The high progression rate of HPV-L1-negative mild and moderate dysplasia emphasizes the precancerous nature of these lesions. A close follow-up with colposcopy and histological evaluation is advisable and removal of these lesions should be considered. The low malignant potential of HPV-L1-positive cases, however, indicates transient HPV infection, justifying a watch and wait strategy with cytological follow-up, thus preventing overtreatment especially for women in their reproductive age. Modern Pathology (2013) 26, 967-974; doi:10.1038/modpathol.2012.233; published online 15 February 2013

Keywords: cervical cancer; HPV L1; prognostic biomarker

Correspondence: Dr G Mehlhorn MD, Department of Obstetrics and Gynecology, Universityhospital Erlangen, Universitätsstrasse 21-23, Erlangen 91054, Germany.

E-mail: grit.mehlhorn@uk-erlangen.de

*Present address: University Hospital Graz, Institute of Cytology, Austria

**Present address: Department of Pathology and Cytodiagnostics, Laboratory Medicine Cologne, Cologne, Germany

Received 6 August 2012; revised 19 December 2012; accepted 20

December 2012; published online 15 February 2013
The benefits of cervical cancer screening programs in reducing morbidity and mortality are well accepted. ${ }^{1}$ On the other hand the harms related to the benefits in particular overtreatment of fertile women only recently started to receive appropriate attention. ${ }^{2,3}$

One of the major problems of cervical cancer screening programs is the lack of an objective test to distinguish a transient human papillomavirus (HPV) infection from true precancer among HPV high-risk positive early dysplastic lesions. 
As a consequence invasive procedures such as colposcopy, biopsy and conisations are being performed too frequently, with high costs and potentially negative impact on reproductive outcomes, including preterm delivery and low-birth-weight infants with related risks for disabilities. ${ }^{4}$

To improve women's and children's quality of life and save health care resources it would be most useful to have prognostic markers to distinguish patients who will experience progression of an early precursor lesion to cancer from those who will not. ${ }^{5}$

Data from the ASCUS-LSIL Triage Study confirmed that HPV high-risk DNA testing is not a useful triage strategy in low-grade SIL (LSIL) cases, ${ }^{6}$ and that it is still unpredictable by morphology alone whether CIN2 represents histological low-grade or high-grade lesion. ${ }^{7}$ Therefore, to optimize the clinical management specific tests are needed to identify women who are at risk for progressive lesions. ${ }^{8}$

Even with the vaccination era rising, HPV will remain a major global health burden. Worldwide Cervical cancer is the third most common cancer in women, and the seventh overall, with an estimated 530000 new cases in 2008. Northern America (USA, Canada) and Europe contributes 75000 of these cases and medical follow-up is recommended for $\sim 7$ million women with abnormal Pap smears. ${ }^{9}$

Different reporting systems are used for Pap smear diagnosis. Besides the original WHO classification, ${ }^{10}$ The Bethesda System ${ }^{11}$ is internationally accepted for cervical cytology. In Germany, the Munich Nomenclature II is being recommended, ${ }^{12}$ which puts mild and moderate dysplasia in the group IIID with recommendation for active surveillance including cytological follow-up and colposcopy.

Germany's watchfull waiting strategy offers the opportunity to investigate the clinical outcome of cytologically detected moderate dysplasia, the cytological equivalent of CIN2. This is being considered a reasonable approach as, independent of the reporting system, only $10 \%$ of the mild and $20 \%$ of the moderate dysplastic lesions develop CIN3 + lesions, but most of them regress spontaneously. ${ }^{13,14}$

Despite this conservative recommendation in the Munich Nomenclature, conisation was one of the most common surgical procedures performed in women of fertile age in Germany during 2010, accounting for more than 50000 cases. ${ }^{15}$

With the introduction of the prophylactic HPV vaccines the immunological properties of the L1 capsid protein, one of eight known HPV-specific proteins, has been intensively investigated mainly focusing on antibody levels and their protective properties.

In addition, several initial retrospective and prospective singlecenter studies with smaller sample sizes and shorter follow-up periodes in Germany, Denmark, Italy and South Korea used HPV L1 capsid protein detection with Cytoactiv to predict the clinical outcome of CIN1 lesions and early dysplastic lesions in combination with p16 or HPV high-risk DNA tests. ${ }^{16-22}$

\section{Materials and methods}

\section{Patients and Design}

For this prospective international multicenter study six centers located in Basel (Switzerland), Bolzano (Italy), Cologne, Einbeck, Erlangen and Salzgitter Bad (Germany) recruited in 2007 randomly selected cases of HIV negative, non-pregnant, non HPV L1 vaccinated women reported as LSIL or moderate (HSIL) dysplasia.

The Pap smears were independently classified by two local investigators and a centralized review of all cases was performed by a reference cytopathologist, HG.

For conventional Pap smears the cells were transferred onto the glass slides and the remaining cells within the brush were used for HPV DNA analysis. For the preparation of ThinPrep and SurePath slides the brush was transferred into the corresponding preservative fluid. After the preparation of the slides for Pap smear diagnosis, the residual cells within the vials were used for HPV DNA analysis.

HPV high-risk association was confirmed with the Hybrid Capture II test (Digene/Qiagen, Hilden, Germany) or a PCR using the MY09/MY11 and $\mathrm{GP} 5+/ \mathrm{GP} 6+$ primer sets. ${ }^{23}$ The amplification products were confirmed by DNA sequencing.

The study protocol was approved by the institutional review board of the University Hospital Erlangen (ethics committee/number 3771). Written informed consent from the patients was not necessary as the samples were received and analyzed anonymously and patients were treated in accordance to the national guidelines in Germany, Switzerland and Italy with cytological follow-up and colposcopy and/or punch biopsies for histological verification.

Follow-up smears were taken in intervals of 3-6 month or annually, after the first negative smear for intraepithelial lesions. A conisation for treatment was performed if clinically indicated, and with the patient's consent.

Owing to the presumed critical importance of immunological reactions any invasive procedure that may be able to induce an immunological reaction was defined as the clinical end point of the study case.

Follow-up ended in June 2011, resulting in a follow-up period of up to 54 month.

Women having at least two consecutive smears negative for intraepithelial lesion were considered to be in clinical remission. Persistence was defined as the state in which mild and moderate dysplasia persisted cytologically over the whole follow-up period or as histology confirmed CIN1/CIN2.

Progression was defined as a histologically confirmed CIN3 + lesion. Histological diagnoses were from conisation specimens or colposcopically guided punch biopsies.

Fisher's exact test, two-sided was used for data analysis. 
Table 1 Inclusion and exclusion number of enrolled patients

\begin{tabular}{|c|c|c|c|}
\hline \multirow{3}{*}{ Total } & \multicolumn{2}{|c|}{$\begin{array}{c}\text { PAP-smear results and } \\
\text { immunocytochemistry of } \\
\text { HPV L1 }(\mathrm{n}=908)\end{array}$} & \multirow{3}{*}{$\mathrm{P}$-value } \\
\hline & $\operatorname{LSIL}(\mathrm{n}=515)$ & HSIL $(\mathrm{n}=393)$ & \\
\hline & $n(\%)$ & $n(\%)$ & \\
\hline Inclusion & 479 & 322 & \multirow{6}{*}{$<0.0001$} \\
\hline L1+ & $328(69 \%)$ & $143(44 \%)$ & \\
\hline L1 - & $151(31 \%)$ & $179(56 \%)$ & \\
\hline Loss of follow-up & 36 & 71 & \\
\hline $\mathrm{L} 1+$ & 26 & 41 & \\
\hline L1- & 10 & 30 & \\
\hline
\end{tabular}

\section{Immunocytochemistry}

Routinely processed conventional Pap smears, ThinPrep and SurePath slides were immunochemically stained with the Cytoactiv Screening Set (Cytoimmun Diagnostics GmbH, Pirmasens, Germany), which detects the L1 capsid protein of all known HPV types. Staining was performed according to manufacturer's protocol, described previously. ${ }^{19}$

In brief, slides used for the initial morphological diagnosis were subjected to antigen unmasking by microwave treatment after unmounting without prior destaining. Cytoactiv screening antibody was applied onto the slides and incubated for $30 \mathrm{~min}$ at room temperature, followed by incubation with the detection reagent for $10 \mathrm{~min}$ and AEC chromogen for 5 min.

After counterstaining with hematoxylin, slides were mounted with Aquatex (Merck, Darmstadt, Germany) and coverslipped.

Stained slides were studied by light microscopy independently by two local investigators and a reference cytopathologist, HG. Slides with at least one epithelial cell with distinctly positive nuclear staining were scored as positive.

\section{Results}

A total of 908 HPV high-risk-associated early dysplastic lesions were recruited for the study, 515 $(57 \%)$ were classified as LSIL, $393(43 \%)$ as HSIL (moderate dysplasia only). Overall, 107 (41 HSIL L1 +, 30 HSIL L1 - , 26 LSIL L1 +, 10 LSIL L1 -) out of 908 cases $(12 \%)$ were lost during the followup period of 54 month (see Table 1).

For data analysis, 801 cases with complete clinical records were included (see Table 2). Mean age of all 801 patients was 33.6 years (range 15-83 years). 33.6 years was the mean age of the 479 LSIL (range 15-83y, L1 - 33.4y, L1 + 33.7y), 322 HSIL (range 16-82y, L1 - 33.7y, L1 + 33.3y), 471 L1positive (range 15-83y) and 330 L1-negative cases (range 16-82y) as well (see Figure 1).
Table 2 Clinical outcome of HPV high-risk positive early dysplastic lesions in relation to the result of immunocytochemistry for L1 protein

$\begin{array}{lcccc}\begin{array}{l}\text { HPV L1 result } \\ \text { Smear (LSIL/ }\end{array} & \begin{array}{c}\text { Remission } \\ \mathrm{n}(\%)\end{array} & \begin{array}{c}\text { Persistance } \\ \mathrm{n}(\%)\end{array} & \begin{array}{c}\text { Progression } \\ \mathrm{n}(\%)\end{array} & \text { P-value* } \\ \text { range) }\end{array}$

*Fisher's exact test.

Fifty-three percent (253/479) of the LSIL cases showed a spontaneous clinical remission, defined as minimum two consecutive negative Pap smears.

Twenty-five percent (118/479) persisted as histologically confirmed CIN1 (60/118) or CIN2 (47/118) and 11 cases persisted cytologically over the whole follow-up period (9 L1-positive LSIL, 2 L1-negative LSIL). Twenty-three percent (108/479) progressed to CIN3 + .

Applying the same criteria for the clinical outcome, we found for the HSIL cases a clinical remission in $25 \%(81 / 322)$, a persistence in $26 \%$ (83/322, 11 CIN1, 71 CIN2, cytologically as $1 \mathrm{~L} 1+$ HSIL) and a progression in $49 \%(158 / 322)$.

Eighty-four percent (68/75) of the cases tested for HPV DNA at remission were HPV negative.

The remaining 11 cases have been HPV DNA positive. As the HPV DNA testing was not performed at any visit, it was not possible to determine whether the HPV DNA persisted or whether a new HPV infection has occurred. The mean number of negative Pap smears for these HPV high-risk positive cases with a clinical remission was 5.5 (range 3-7).

According to the previously established cutoff value all slides with at least one epithelial cell with distinctly positive nuclear staining were scored as positive. Examples for L1 positive and negative LSIL and HSIL are shown on Figures 2a and b, 3a and b. The observed agreement and kappa for HPV L1 immunostaining in our study were 98\% and 0.96 (95\% confidence interval: 0.89-1.00) respectively.

Detection rate of the L1 capsid protein was $69 \%$ (328/479) for LSIL and 44\% (143/322) for HSIL cases (Table 1).

The difference of the clinical outcome of the L1-negative cases and the L1-positive cases was 


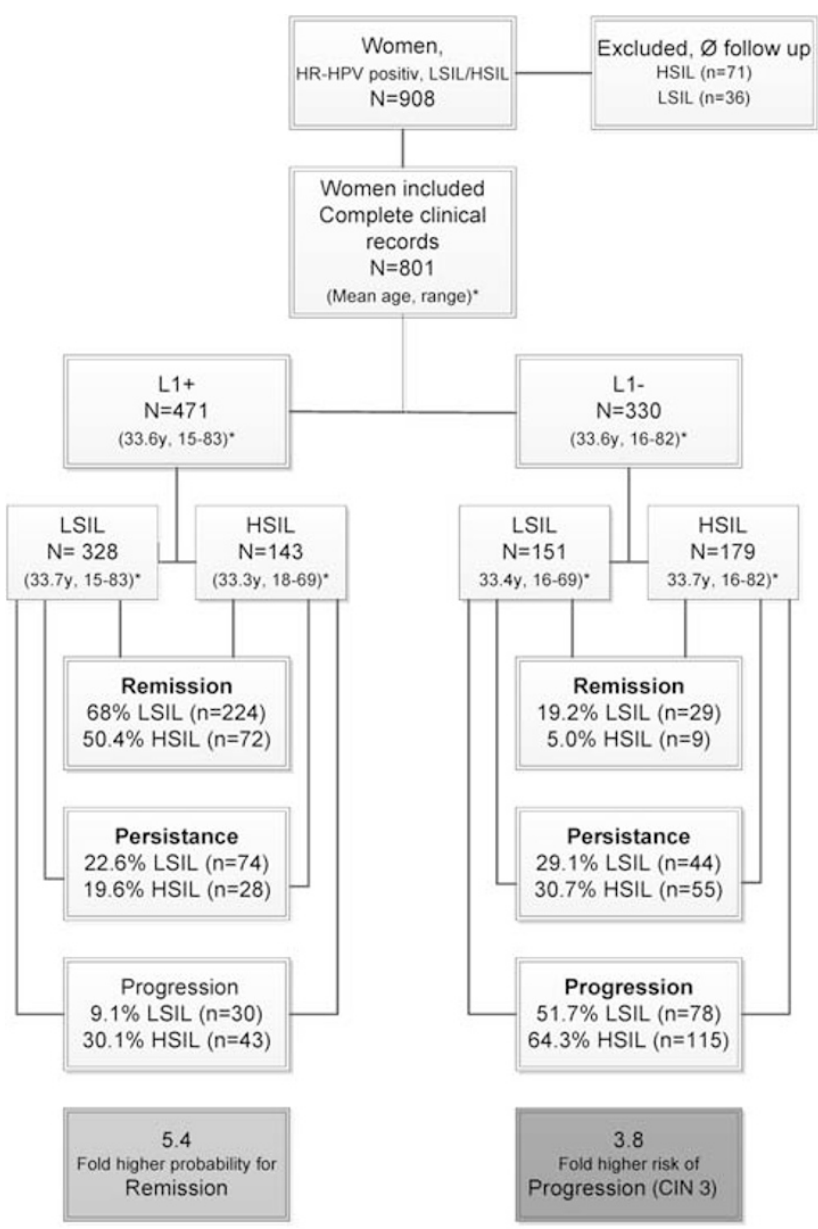

Figure 1 Participant flow diagram and clinical outcome of HPV high-risk positive early dysplastic lesions in relation to the result of immunocytochemistry for L1 protein.

statistically highly significant $(P$-value $<0.0001)$ and independent of the classification of LSIL (mild dysplasia) and HSIL (moderate dysplasia), see Table 2.

A total of $296(63 \%)$ out of 471 L1-positive LSIL and HSIL but only $38(12 \%)$ out of 330 L1-negative cases showed a spontaneous clinical remission of the lesion. This is a 5.4-fold higher probability for clinical remission of the L1-positive cases compared with the L1-negative cases.

In accordance with these findings, only $73(16 \%)$ out of 471 L1-positive but 193 (59\%) out of 330 L1-negative cases progressed to CIN3 +, a 3.8-fold higher risk of progression for L1-negative cases.

The rate of clinical remission was highest among the L1-positive LSIL (68\%, 224/328 cases) followed by L1-positive HSIL cases $(50 \%, 72 / 143)$ and lowest within the L1-negative LSIL $(19 \%, 29 / 151)$ and the L1-negative HSIL (5\%, 9/179).

In accordance with these findings the rate of progression was lowest within the L1-positive LSIL $(9 \%, 30 / 328)$ the L1-positive HSIL $(30 \%, 43 / 143)$ and highest for the L1-negative LSIL (52\%, 78/151), and the L1-negative HSIL (64\%, 115/179).
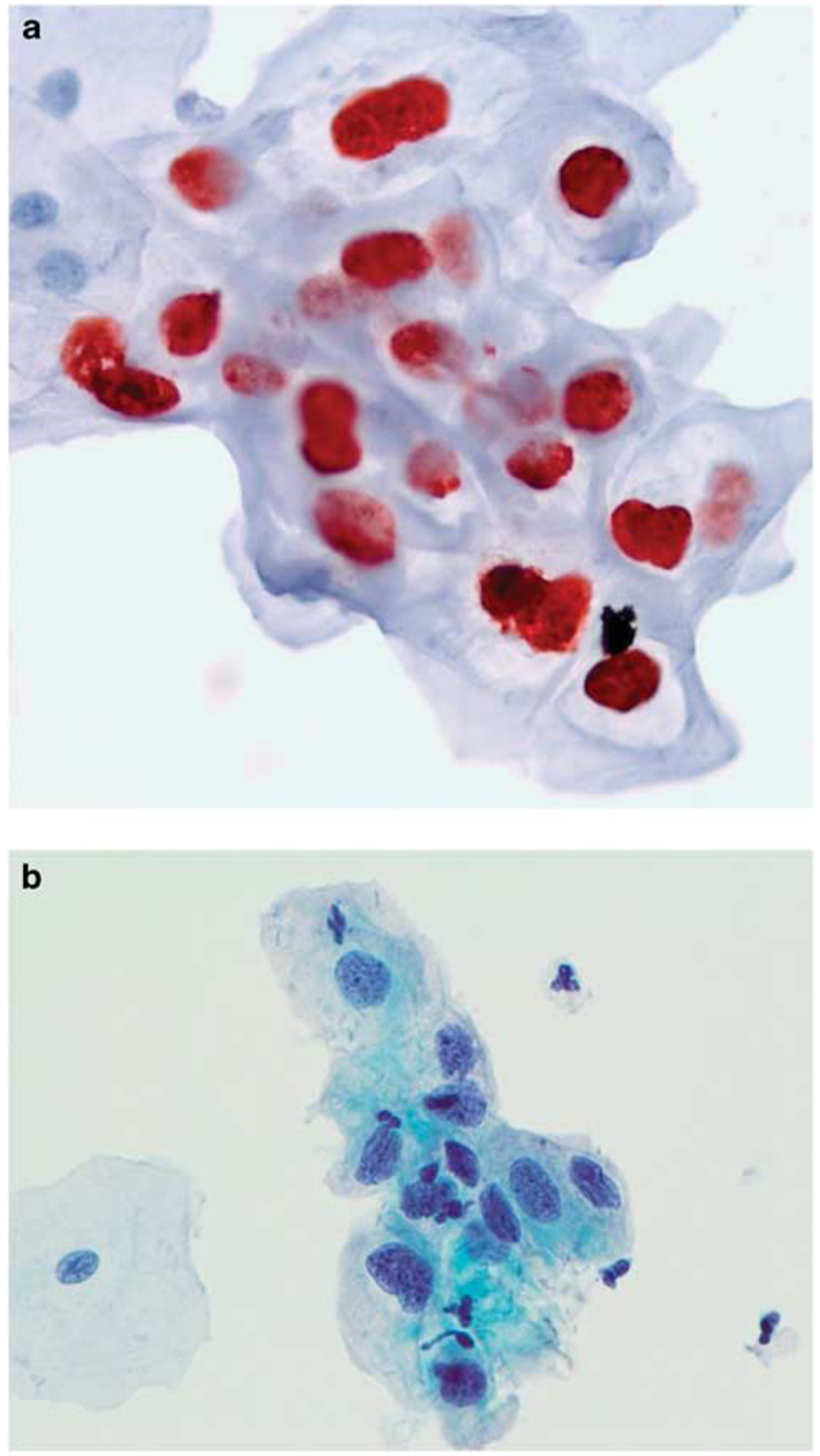

Figure 2 (a) L1 capsid protein positve low-grade squamous intraepithelial lesion, showing a strong nuclear staining. (b) L1 capsid protein negative low-grade squamous intraepithelial lesion.

That means that L1-negative LSIL cases had a 5.6 times higher risk of progression than the L1-positive LSIL and L1-positive HSIL cases had a 9.9-fold higher probability of clinical remission compared with the L1-negative HSIL cases.

Interestingly, the HSIL cases expressing the L1 capsid protein $(50 \%)$ have a 2.6 -fold higher probability to regress and a 1.7-fold lower risk of progression than the LSIL being L1 capsid protein negative (19\%, $P<0.0001)$.

Stable disease was found in $102(22 \%)$ of the 471 L1-positive and $99(30 \%)$ of the 330 L1-negative cases. The percentage of stable disease was independent of the L1-positive/negative status and the LSIL/HSIL classification and ranged from 20 to $31 \%$. A detailed description is given in Table 3.

The different recommendations taking colposcopically guided punch biopsies for histological 
Table 3 Cases defined as 'persistence'/'stable disease'

\begin{tabular}{|c|c|c|c|c|}
\hline HPV L1 result/ & Persistence & $\begin{array}{l}\text { Punch } \\
\text { biopsy }\end{array}$ & Cytological & $\begin{array}{l}\text { Cone } \\
\text { biopsy }\end{array}$ \\
\hline $\begin{array}{l}\text { Smear (LSIL/ } \\
\text { HSIL) }\end{array}$ & $\begin{array}{l}\text { No. of } \\
\text { cases }\end{array}$ & $\begin{array}{l}\text { No. of } \\
\text { cases }\end{array}$ & $\begin{array}{l}\text { No. of } \\
\text { cases }\end{array}$ & $\begin{array}{l}\text { No. of } \\
\text { cases }\end{array}$ \\
\hline \multirow[t]{2}{*}{ L1 + LSIL 74} & $\begin{array}{l}49 \text { CIN1/ } \\
\text { LSIL }\end{array}$ & 34 & 9 & 6 \\
\hline & 25 CIN2 & 14 & 0 & 11 \\
\hline \multirow[t]{2}{*}{ L1 - LSIL 44} & $\begin{array}{l}22 \text { CIN1/ } \\
\text { LSIL }\end{array}$ & 9 & 2 & 11 \\
\hline & 22 CIN2 & 3 & 0 & 19 \\
\hline \multirow[t]{2}{*}{ L1 + HSIL 28} & 2 CIN1 & 0 & 0 & 2 \\
\hline & $\begin{array}{l}26 \text { CIN2/ } \\
\text { HSIL }\end{array}$ & 7 & 1 & 18 \\
\hline \multirow[t]{2}{*}{ L1 - HSIL 55} & 9 CIN1 & 1 & 0 & 8 \\
\hline & $\begin{array}{l}46 \text { CIN2/ } \\
\text { HSIL }\end{array}$ & 11 & 0 & 35 \\
\hline
\end{tabular}

verification of abnormal Pap became obvious as one study center contributed $46(62 \%)$ out of 74 cases of stable disease for L1-positive LSIL.

As any invasive procedure may be able to influence the natural outcome of the LSIL and HSIL cases this was defined as the clinical end point of a study case.

Therefore, we calculated the data without these stable disease cases as well. This projection sharpens the difference in the malignant potential of L1-positive and L1-negative cases. L1-negative cases have a high malignant potential. Eighty-four percent $(193 / 231)$ progressed to CIN3 +, whereas $80 \%$ (296/369) of the L1-positive cases showed a clinical remission. With $20 \%$ the risk of progression for L1positive cases is low (Figure 4).

\section{Discussion}

Since the early days of cervical cytology, it is known that the morphologically identified lesions of different grades are mixtures of distinct biological stages resulting in different clinical outcomes, remission or progression. ${ }^{24}$

Today, with the knowledge that HPV is a necessary but not sufficient cause for the development of most cervical cancers, transient HPV infection and precancer are often used synonymous for these biologically different conditions.

In view of the fact that both, morphology and HPV high-risk DNA testing, are unable to predict clinical remission or disease progression, ${ }^{25}$ diagnosis of CIN2 or worse is the clinical threshold leading to ablative or excisional therapy in the United States and Europe.

The study reported reflects a common screening policy in several European countries, recommending not a two tier classification like The Bethesda System, but a three tier classification (the Munich nomenclature II) reporting mild dysplasia, moderate dysplasia and severe dysplasia as distinct morphological categories. Mild dysplasias, together with
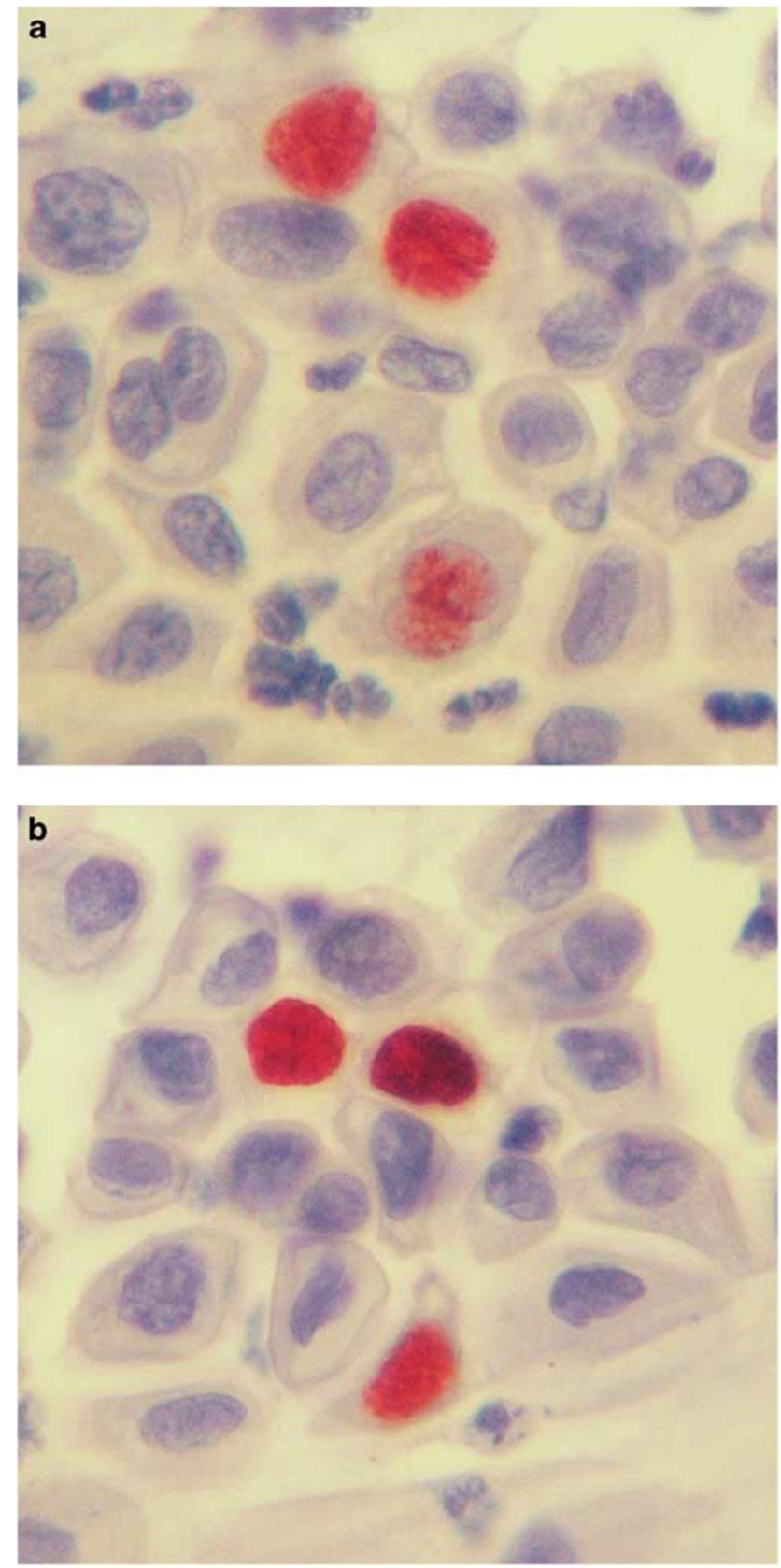

Figure 3 (a, b). L1 capsid protein positve high-grade squamous intraepithelial lesion, showing a strong, nuclear staining.

moderate dysplasias are grouped in category Group IIID. A cytological follow-up of these women is recommended. Generally, only in cases of persistent mild or moderate dysplasia a colposcopical evaluation including punch biopsy is performed at specialized centers. The 'cutoff' point for mandatory histological evaluation is set between moderate dysplasia and severe dysplasia.

Even with a watch and wait strategy for moderate dysplasia, the cytological CIN2 equivalent, every year more than 50000 women of fertile age are treated by a conization in Germany, which is one of the most common surgical therapies performed in 


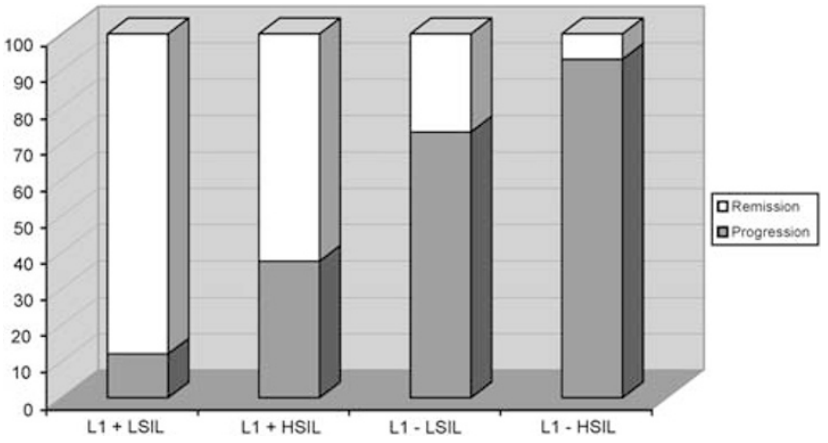

Figure 4 L1 capsid protein detection and the malignant potential for abnormal smears. Risk profiles for remission and progression depending on the L1 status and the severity of the lesion.

this age group. The cumulative costs for each patient are calculated with $2178 € .^{15}$ That means that only in Germany 110 Million Euro are spent annually for the therapies of these women. Most probably in the USA and the rest of Europe the number and total costs are 10 times higher.

To prevent overtreatment and to reduce the overtreatment-related costs an objective standard is missing that stratifies treatment according to the individual risk profile of women and to overcome patient management based on average risk profiles defined by morphology groups and age.

This 'non invasive' cytological follow-up strategy offers the unique possibility to investigate the natural outcome of HPV high-risk associated moderate dysplasia.

The critical importance of cell-mediated immune responses in the resolution and control of HPV infections is well known. On the one hand, infiltrating $\mathrm{T}$ lymphocytes and macrophages can be observed in spontaneously regressing papillomas, and on the other hand, increased incidence and progression of HPV infections in immunosuppressed patients are obvious.

Therefore, any invasive procedure that may be able to trigger the immune response, such as colposcopically guided punch biopsies induced wound healing processes or release of endogenous cellular or viral antigens was defined as a 'non natural' condition and the clinical end point of the study case.

Morphological diagnoses are subject of interobserver interpretative variability and this is especially true for moderate dysplasia (CIN2/HSIL moderate).

To classify LSIL and HSIL moderate correctly and consistently the study protocol recommended the independent classification of two local investigators and an additional centralized review of all cases.

For the sake of diagnostic specificity, only $\geq$ CIN3, a substantially more reproducible diagnosis than CIN2, ${ }^{26}$ was considered for progression and CIN2 as stable disease although the authors are well aware that some of the CIN2 biologically behave as CIN3. This may be a confounding variable of our study compared with others.
In our study the complete clinical records of 479 HPV high-risk positive LSIL and 322 HPV high-risk positive HSIL are reported. Twenty-three percent of the LSIL and $49 \%$ of the HSIL progressed to CIN3 +, according to Ostor risk profiles that typically could be expected for these kinds of early dysplastic lesions.

The Munich Nomenclature II being used in Germany recommends a cytological follow-up and colposcopy for these 322 HSIL rather than treatment, whereas the clinical threshold for treatment, primarily by excision, according to the Bethesda System is CIN2.

This study shows that HPV-L1 capsid protein analysis with Cytoactiv is able to predict the clinical outcome of early dysplastic lesions, helps to discriminate transient HPV infection from HPVinduced precancer and could be used as an objective test to optimize the clinical management of early squamous intraepithelial lesions.

After the viral replication has taken place the intermediate cells start to produce the L1 protein and the protein is constantly accumulated within the nucleus until the cells are approaching the superficial layer. The typical L1 staining is a strong, homogenous nuclear stain being the reason for the observed very good interobserver reproducibility of $98 \%$.

The Kappa value of 0.96 (95\% confidence interval 0.89-1.00) according to Landis and $\mathrm{Koch}^{27}$ has to be classified as 'almost perfect'. Using histological sections Galgano et al. ${ }^{28}$ reported for Cytoactiv quite similar results with a raw agreement and $\mathrm{k}$ of $97 \%$ and $0.88 \%$, respectively.

Terminal differentiation of the basal epithelial host cell is essential for HPV L1 synthesis and free viral particles are released from the L1 capsid protein positive apical layers of keratinocytes to infect other cells and hosts. ${ }^{29}$

L1 capsid protein negative cases, however, have lost the capability to produce virions because of the disrupted squamous epithelial differentiation due to virally induced cellular deregulation.

Absence of L1 capsid protein in squamous intraepithelial lesion allows the virus to evade the innate and to delay the activation of the adaptive immune response, whereas L1-positive squamous intraepithelial lesion is eliminated by the immune system.

Free viral particles consisting of 360 L1 capsid proteins are the only fully accessible antigen sources in the earlier stage of virally induced squamous intraepithelial lesion to promote an activation of the immune system.

To generate an effective virus-specific immune response the virus particles have to be detected by the antigen-presenting cells of squamous epithelium, the Langerhans cells or dendritic cells, and armed effector cells, has to migrate back to the infected site, and destroy the infected keratinocytes leading to a spontaneous clinical remission of the lesion. ${ }^{30}$

If such immunologic activation mechanisms are functional, they are quite effective as with $20 \%$ the malignant potential for these L1-positive lesions, 
irrespective of the dysplasia being cytologically mild or moderate, is low.

Using smaller sample sizes and shorter follow-up times similar results were reported from different authors using cytology and biopsy specimens. ${ }^{16-22}$

An ineffective immune response may be promoted by factors contributing to cervical cancer such as tobacco smoking or the coexistence of dysplasias of different grades (CIN1/CIN2) in the transformation zone, possibly reflecting a mixture of L1-positive and L1-negative lesions with different progressive potentials may be the reason for a progression of some of these L1-positive intraepithelial lesions.

On the other hand, with $84 \%$ the malignant potential of the L1-negative cases is exceedingly high, similar to what is expected for a true precancerous lesion. The differentiation-dependent loss of the L1 stimulus may lead to a local 'lack of immunity' further supporting the virally induced alterations. These may lead to additional disorders of cell cycle regulation at transcriptional, translational and genomic levels, thus resulting in a progression of the early precursor lesions to CIN3.$+{ }^{31-33}$

Only $5 \%$ of the L1-negative HSIL and $19 \%$ of the L1-negative LSIL showed a spontaneous remission.

Reasons for the clinical remission of L1-negative cases are most likely sampling errors with absence of L1-expressing cells in the sample or expression levels below the detection limit of the immunochemical assay.

Therefore, an additional confirmation of the L1 status in the biopsy specimen may be helpful in some of these cases. On the other hand, the L1negative status is in most cases the 'true feature of the lesion' and apparently not a sampling issue of the Pap smear. ${ }^{22}$

Negri and colleagues ${ }^{18}$ reported for coexisting CIN1/CIN3 lesions, that all of the CIN3 and about $70 \%$ of the coexisting CIN1 lesions were already L1-negative. It seems that this loss of L1 capsid protein synthesis is an early precancerous event, and CIN 3 lesions may evolve out of such L1negative CIN1 lesions.

Using our data the clinical management of women with early dysplastic lesions could be improved recommending treatment according to the individual risk profile of the women rather than generalizing risk groups because of a similar morphology as it is generally done.

The risk of progression to CIN3 + was 5.6-fold higher for L1-negative LSIL than for the L1-positive LSIL.

Even more impressively, L1-positive HSIL cases had a 9.9-fold higher chance to regress than L1-negative HSIL. This shows that LSIL and HSIL are mixtures of distinct biological stages and that morphology by itself does not predict which lesion will regress or progress.

Interestingly, and not correlating with the clinical recommendations so far, with $73 \%$ LSIL being
L1-negative have a much higher risk of progression to CIN3 + than HSIL being L1 positive (37\%).

Taking this data into account it seems reasonable to call L1-positive HSIL, as lesions with a low malignant potential, together with the L1-positive LSIL, low-grade lesion or transient HPV infection. A watch and wait strategy with follow-up smears within the next 6-12 month plus a control of the L1 status rather than ablative or excisional procedures should be advised.

L1-negative HSIL as precancer warrants immediate treatment as $<10 \%$ regressed spontaneously over the follow-up period of 54 month.

Owing to the risk profiles L1-negative LSIL should be considered precancer as well. This could lead to a novel approach for clinical management of LSIL cases.

So far, low-grade dysplastic lesions were generally considered to have a low risk for development of CIN3 + . Our data show, however, that L1-negative LSIL have a high potential for progression. Over 70\% of these lesions progressed to CIN3 + , which indicates that the usual follow-up strategy for such cytologically low-grade lesions might not be appropriate.

In summary, to maximize the benefits and reduce the costs and risks of overtreatment of Cervical Cancer Screening programs, we suggest the following strategy for HPV high-risk positive mild or moderate dysplastic lesions:

(1) L1-negative dysplasias have a high malignant potential and need a close follow-up with colposcopy and histological evaluation.

(2) L1-positive mild and moderate dysplasias are true low-grade lesions in which a wait and watch strategy with cytological follow-up within 6-12 months is justified. Only in case of persistence of the lesion a colposcopy should be performed.

\section{Disclosure/conflict of interest}

Dr Hilfrich works as an employee for Cytoimmun Diagnostics $\mathrm{GmbH}$. The remaining authors declare no conflict of interest.

\section{References}

1 Johannesson G, Geirsson G, Day N. The effect of mass screening in Iceland, 1965-74, on the incidence and mortality of cervical carcinoma. Int J Cancer 1978;21: 418-425.

2 Sadler L, Saftlas A, Wang W, et al. Treatment for cervical intraepithelial neoplasia and risk of preterm delivery. JAMA 2004;291:2100-2106.

3 Bevis KS, Biggio JR. Cervical conization and the risk of preterm delivery. Am J Obstet Gynecol 2011;205: 19-27.

4 Kyrgiou M, Koliopoulos G, Martin-Hirsch P, et al. Obstetric outcomes after conservative treatment for 
intraepithelial or early invasive cervical lesions: systematic review and meta-analysis. Lancet 2006;367: 489-498.

5 Nucci MR, Castrillon DH, Bai H, et al. Biomarkers in diagnostic obstetrics and gynaecological pathology: a review. Adv Anat Pathol 2003;10:55-68.

6 The ALTS Group. Human papillomavirus testing for triage of women with cytological evidence of low grade squamous intraepithelial lesions: baseline data from a randomized trial. J Natl Cancer Inst 2006;92:397-402.

7 Castle PE, Schiffman M, Wheeler CM, et al. Evidence for frequent regression of cervical intraepithelial neoplasia-grade. Obstet Gynecol 2009;113:18-25.

8 Castle PE, Stoler MH, Solomon D, et al. The Relationship of community biopsy-diagnosed cervical intraepithelial neoplasia grade 2 to the quality controlreviewed diagnosis. Am J Clin Pathol 2007;127:805-815.

9 Ferlay J, Shin HR, Bray F, et al. GLOBOCAN 2008 v1.2, Cancer Incidence and Mortality Worldwide: IARC Cancer Base No. 10. Lyon, France: International Agency for Research on Cancer 2010. available from: http://globocan.iarc.fr.

10 Riotton G, Christopherson WM, Lunt R. Cytology of the Female Genital Tract. International Histological Classification of Tumours No. 8. World Health Organisation: Geneva, 1973.

11 Solomon D, Davey D, Kurman R, et al. The 2001 Bethesda System: terminology for reporting results of cervical cytology. JAMA 2002;287:2114-2119.

12 Wagner D. Munich nomenclature II for gynaecologic cytodiagnosis. Acta Cytol 1990;34:900-901.

13 Cantor SB, Atkinson EN, Cardenas-Turanzas M, et al. Natural History of Cervical Intraepithelial Neoplasia. Acta Cytol 2005;49:405-415.

14 Ostor A. Natural history of cervical intraepithelial neoplasia: a critical review. Int J Gynecol Pathol 1993; 12:186-192.

15 Soergel P, Makowski E, Makowski L, et al. What are the costs of conisation when considering pregnancy associated complications? Geb Fra 2011;71:199-204.

16 Rauber D, Mehlhorn G, Fasching PA, et al. Prognostic significance of the detection of the human papillomavirus L1 protein in smears of mild to moderate cervical intraepithelial lesions. Eur J Obstet Gynecol Reprod Biol 2008;140:258-262.

17 Hilfrich R, Hariri J. Prognostic relevance of HPV L1 capsid protein detection within mild to moderate dysplastic lesions of the cervix uteri in combination with a second biomarker p16. Anal Quant Cytol Histol 2008;30:78-82.

18 Negri G, Bellisano G, Zannoni GF, et al. p16 and HPV immunohistochemistry is helpful for estimating the behaviour of low grade dysplastic lesions of the cervix uteri. Am J Surg Pathol 2008;32:1715-1720.
19 Griesser H, Sander H, Walczak C, et al. HPV vaccine protein L1 predicts disease outcome of high-risk $\mathrm{HPV}+$ early dysplastic lesions. Am J Clin Pathol 2009;132:840-845.

20 Choi YS, Kang WD, Kim SM, et al. Human papillomavirus L1 capsid protein and human papillomavirus type 16 as prognostic markers in cervical intraepithelial neoplasia 1. Int J Gynecol Cancer 2010; 20:288-293.

21 Lee SJ, Lee AW, Kim TJ, et al. Correlation between immunocytochemistry of human papilloma virus L1 capsid protein and behavior of low-grade cervical cytology in Korean women. J Obstet Gynaecol Res 2011;37:1222-1228.

22 Lee H, Lee KJ, Jung CK, et al. Expression of HPV L1 capsid protein in cervical specimens with HPV infection. Diagn Cytopathol 2008;36:864-867.

$23 \mathrm{Qu} \mathrm{W}$, Jiang G, Cruz Y, et al. PCR detection of human papillomavirus: comparison between MY09/MY11 and GP5 + /GP6 + primer systems. J Clin Microbiol 1997;35:1304-1310.

24 Papanicolaou GN, Traut HF. Diagnosis of uterine cancer by the vaginal smear. Commonwealth Fund: New York, 1943.

25 ASCUS-LSIL Traige Study (ALTS) Group. A randomized trial on the management of low-grade squamous intraepithelial lesion cytology interpretations. Am J Obstet Gynecol 2003;188:1393-1400.

26 Carreon JD, Sherman ME, Guillen D, et al. CIN2 is a much less reproducible and less valid diagnosis than CIN3: results from a histological review of population based cervical samples. Int J Gynecol Pathol 2007; 26:441-446.

27 Landis JR, Koch GG. The measurement of observer agreement for categorical data. Biometrics 1977;33: 159-174.

28 Galgano MT, PhE Castle, Atkins KA, et al. Using biomarkers as objective standards in the diagnosis of cervical biopsies. Am J Surg Pathol 2010;34:1077-1087.

29 Doorbar J. Papillomavirus life cycle organization and biomarker selection. Dis Markers 2007;23:297-313.

30 Stanley M. Immune response to human papillomavirus. Vaccine 2006;24:16-22.

31 Choo KB, Lee HH, Pan CC, et al. Sequence duplication and internal deletion in the integrated human papillomavirus type 16 genome from cervical carcinoma. J Virol 1998;62:1659-1666.

32 Icenogle JP, Clancy KA, Lin SY. Sequence variation in the capsid protein genes of human papillomavirus type 16 and type 31. Virology 1995;214:664-669.

33 Yang R, Wheeler CM, Chen X, et al. Papillomavirus capsid mutation to escape dentritic cell dependent innate immunity in cervical cancer. J Virol 2005; 79:6741-6750. 